\title{
A New Generalization on Cauchy-Schwarz Inequality
}

\author{
Songting Yin \\ Department of Mathematics and Computer Science, Tongling University, Tongling, Anhui 244000, China
}

Correspondence should be addressed to Songting Yin; yst419@163.com

Received 7 April 2017; Accepted 23 May 2017; Published 13 June 2017

Academic Editor: Yoshihiro Sawano

Copyright (C) 2017 Songting Yin. This is an open access article distributed under the Creative Commons Attribution License, which permits unrestricted use, distribution, and reproduction in any medium, provided the original work is properly cited.

We extend the well-known Cauchy-Schwarz inequality involving any number of real or complex functions and also give a necessary and sufficient condition for the equality. This is another generalized version of the Cauchy-Schwarz inequality.

\section{Introduction}

Let $a=\left(a_{1}, a_{2}, \ldots, a_{n}\right)$ and $b=\left(b_{1}, b_{2}, \ldots, b_{n}\right)$ be two vectors in $\mathbb{R}^{n}$. Then the discrete version of Cauchy-Schwarz inequality is (see $[1,2]$ )

$$
\sum_{i=1}^{n} a_{i}^{2} \sum_{i=1}^{n} b_{i}^{2} \geq\left(\sum_{i=1}^{n} a_{i} b_{i}\right)^{2}
$$

and its integral representation in the space of the continuous real-valued functions $C([a, b], \mathbb{R})$ reads (see $[2,3])$

$$
\int_{a}^{b} f(x)^{2} d x \int_{a}^{b} g(x)^{2} d x \geq\left(\int_{a}^{b} f(x) g(x) d x\right)^{2} .
$$

In undergraduate teaching material, the above two inequalities are presented. Some other forms, such as matrix form and determinant form, are shown in many literatures. It is well known that the Cauchy-Schwarz inequality plays an important role in different branches of modern mathematics such as Hilbert space theory, probability and statistics, classical real and complex analysis, numerical analysis, qualitative theory of differential equations, and their applications. Up to now, a large number of generalizations and refinements of the Cauchy-Schwarz inequality have been investigated in the literatures (see $[4,5]$ ). In [6], Harvey generalized it to an inequality involving four vectors. Namely, for any $a, b, c, d \in$ $\mathbb{R}^{n}$, it holds that

$$
\begin{gathered}
\|a\|^{2}\|b\|^{2}+\|c\|^{2}\|d\|^{2} \geq \\
2 a^{T} c b^{T} d+\left(a^{T} b\right)^{2}+\left(c^{T} d\right)^{2} \\
-\left(a^{T} d\right)^{2}-\left(b^{T} c\right)^{2} .
\end{gathered}
$$

It is a new generalized version of the Cauchy-Schwarz inequality. Recently, the result was refined by Choi [7] to a stronger one:

$$
\begin{aligned}
& \sum_{k=1}^{m}\left\|a_{(k)}\right\|^{2}\left\|b_{(k)}\right\|^{2} \\
& \geq \sum_{k=1}^{m}\left(a_{(k)}^{T} b_{(k)}\right)^{2} \\
& \quad+\frac{2}{m-1} \sum_{1 \leq k<l \leq m}\left|a_{(k)}^{T} a_{(l)} b_{(k)}^{T} b_{(l)}-a_{(k)}^{T} b_{(l)} b_{(k)}^{T} a_{(l)}\right|
\end{aligned}
$$

for any $a_{(k)}, b_{(k)} \in \mathbb{R}^{n}, k=1, \ldots, m$, and $m \geq 2$. Furthermore, he also gained the complex version of the inequality. Since the articles are not so difficult to understand, they are more important for undergraduate students to study.

To meet the need for the teaching, we will consider in this paper the counterparts of (4) and extend them into the following:

$$
\begin{aligned}
& \sum_{k=1}^{m}\left\|f_{k}\right\|^{2}\left\|h_{k}\right\|^{2} \geq \sum_{k=1}^{m}\left\langle f_{k}, h_{k}\right\rangle^{2} \\
& \quad+\frac{2}{m-1} \sum_{1 \leq k<l \leq m}\left|\left\langle f_{k}, f_{l}\right\rangle\left\langle h_{k}, h_{l}\right\rangle-\left\langle f_{k}, h_{l}\right\rangle\left\langle h_{k}, f_{l}\right\rangle\right|
\end{aligned}
$$

for $2 m$ real-valued functions $\left\{f_{k}, h_{k} \in C^{0}([a, b], \mathbb{R}) \mid k=\right.$ $1, \ldots, m, m \geq 2\}$ (Theorem 2 ). Instead of the Euclidean norm 
on $\mathbb{R}^{n}$ or $\mathbb{C}^{n}$ used in $[6,7]$, here the norm and inner product of functions are defined by

$$
\begin{aligned}
\|f\|^{2} & =: \int_{a}^{b} f(x)^{2} d x, \\
\langle f, h\rangle & =: \int_{a}^{b} f(x) h(x) d x .
\end{aligned}
$$

In this work, we further give a necessary and sufficient condition if the equality holds in (5). The complex version of inequality (5) is also obtained (Theorem 4). The whole proof is direct as in $[6,7]$, but the calculations have to be made with some adjustments in the integral case.

\section{The Real Case of the Cauchy-Schwarz Inequality}

Theorem 1. Let $e(x), f(x), g(x)$, and $h(x)$ be four continuous real-valued functions on $[a, b]$. Then

$$
\begin{aligned}
\|e\|^{2} & \|f\|^{2}+\|g\|^{2}\|h\|^{2} \\
\geq & \langle e, f\rangle^{2}+\langle g, h\rangle^{2} \\
& +2|\langle e, g\rangle\langle f, h\rangle-\langle e, h\rangle\langle f, g\rangle|,
\end{aligned}
$$

where the equality holds if and only if $e(x) f(y)-e(y) f(x)=$ $\pm(g(x) h(y)-g(y) h(x))$ for all $x, y \in[a, b]$.

Proof. Notice that $\|e\|^{2}=\int_{a}^{b} e(x)^{2} d x,\|f\|^{2}=\int_{a}^{b} f(x)^{2} d x$ and $\langle e, f\rangle=\int_{a}^{b} e(x) f(x) d x$. Then we have

$$
\begin{aligned}
\|e\|^{2} & \|f\|^{2}-\langle e, f\rangle^{2} \\
= & \int_{a}^{b} e(x)^{2} d x \times \int_{a}^{b} f(x)^{2} d x \\
& -\left(\int_{a}^{b} e(x) f(x) d x\right)^{2} \\
= & \int_{a}^{b} e(x)^{2} d x \int_{a}^{b} f(y)^{2} d y \\
& -\int_{a}^{b} e(x) f(x) d x \int_{a}^{b} e(y) f(y) d y \\
= & \iint_{D}\left[e(x)^{2} f(y)^{2}-e(x) f(x) e(y) f(y)\right] d x d y \\
= & \frac{1}{2} \iint_{D}[e(x) f(y)-e(y) f(x)]^{2} d x d y,
\end{aligned}
$$

where $D=\left\{(x, y) \in \mathbb{R}^{2} \mid a \leq x, y \leq b\right\}$. Using the formula above, we obtain

$$
\begin{aligned}
& \|e\|^{2}\|f\|^{2}+\|g\|^{2}\|h\|^{2}-\langle e, f\rangle^{2}-\langle g, h\rangle^{2}=\frac{1}{2} \\
& \cdot \iint_{D}\left[(e(x) f(y)-e(y) f(x))^{2}+(g(x) h(y)\right. \\
& \left.\quad-g(y) h(x))^{2}\right] d x d y=\frac{1}{2}
\end{aligned}
$$

$$
\begin{aligned}
& \cdot \iint_{D}\{[(e(x) f(y)-e(y) f(x)) \\
& \pm(g(x) h(y)-g(y) h(x))]^{2} \mp 2(e(x) f(y) \\
& -e(y) f(x))(g(x) h(y) \\
& -g(y) h(x))\} d x d y \geq \mp \iint_{D}(e(x) \\
& \cdot f(y)-e(y) f(x))(g(x) h(y)-g(y) \\
& \cdot h(x)) d x d y=\mp 2\left(\int_{a}^{b} e(x) g(x) d x\right. \\
& \cdot \int_{a}^{b} f(y) h(y) d y-\int_{a}^{b} e(x) h(x) d x \int_{a}^{b} f(y) \\
& \cdot g(y) d y)=\mp 2(\langle e, g\rangle\langle f, h\rangle-\langle e, h\rangle\langle f, \\
& g\rangle),
\end{aligned}
$$

which gives the desired inequality. The condition for the equality is obvious. This finishes the proof.

Theorem 2. Let $f_{1}, \ldots, f_{m}, h_{1}, \ldots, h_{m}$ be $2 m$ continuous realvalued functions. Then, for $m \geq 2$, one has

$$
\begin{aligned}
& \sum_{k=1}^{m}\left\|f_{k}\right\|^{2}\left\|h_{k}\right\|^{2} \geq \sum_{k=1}^{m}\left\langle f_{k}, h_{k}\right\rangle^{2}+\frac{2}{m-1} \\
& \cdot \sum_{1 \leq k<l \leq m}\left|\left\langle f_{k}, f_{l}\right\rangle\left\langle h_{k}, h_{l}\right\rangle-\left\langle f_{k}, h_{l}\right\rangle\left\langle h_{k}, f_{l}\right\rangle\right|,
\end{aligned}
$$

where the equality holds if and only if $f_{k}(x) h_{k}(y)-$ $f_{k}(y) h_{k}(x)= \pm\left(f_{l}(x) h_{l}(y)-f_{l}(y) h_{l}(x)\right)$ for all $k, l=1, \ldots, m$ and all $x, y \in[a, b]$.

Proof. It follows from Theorem 1 that

$$
\begin{gathered}
\left\|f_{k}\right\|^{2}\left\|h_{k}\right\|^{2}+\left\|f_{l}\right\|^{2}\left\|h_{l}\right\|^{2}-\left\langle f_{k}, h_{k}\right\rangle^{2}-\left\langle f_{l}, h_{l}\right\rangle^{2} \\
\geq 2\left|\left\langle f_{k}, f_{l}\right\rangle\left\langle h_{k}, h_{l}\right\rangle-\left\langle f_{k}, h_{l}\right\rangle\left\langle h_{k}, f_{l}\right\rangle\right|
\end{gathered}
$$

for $1 \leq k<l \leq m$. Thus, we have

$$
\begin{aligned}
& \sum_{k=1}^{m}\left[\left\|f_{k}\right\|^{2}\left\|h_{k}\right\|^{2}-\left\langle f_{k}, h_{k}\right\rangle^{2}\right]=\frac{1}{m-1} \\
& \cdot \sum_{1 \leq k<l \leq m}\left[\left\|f_{k}\right\|^{2}\left\|h_{k}\right\|^{2}+\left\|f_{l}\right\|^{2}\left\|h_{l}\right\|^{2}-\left\langle f_{k}, h_{k}\right\rangle^{2}\right. \\
& \left.\quad-\left\langle f_{l}, h_{l}\right\rangle^{2}\right] \geq \frac{2}{m-1} \sum_{1 \leq k<l \leq m} \mid\left\langle f_{k}, f_{l}\right\rangle\left\langle h_{k}, h_{l}\right\rangle \\
& \quad-\left\langle f_{k}, h_{l}\right\rangle\left\langle h_{k}, f_{l}\right\rangle \mid .
\end{aligned}
$$




\section{The Complex Case of the Cauchy-Schwarz Inequality}

In this section, we consider the complex case. The norm and the inner product of complex-valued functions are defined by

$$
\begin{aligned}
\|f\|^{2} & =: \int_{a}^{b} f(x) \overline{f(x)} d x, \\
\langle f, h\rangle & =: \int_{a}^{b} f(x) \overline{h(x)} d x, \\
f, h & \in C^{0}([a, b], \mathbb{C}) .
\end{aligned}
$$

First, we give the following.

Theorem 3. Let e, $f, g$, and $h$ be four continuous complexvalued functions on $[a, b]$. Then

$$
\begin{aligned}
& \|e\|^{2}\|f\|^{2}+\|g\|^{2}\|h\|^{2} \\
& \geq|\langle e, f\rangle|^{2}+|\langle g, h\rangle|^{2} \\
& \quad+2|\operatorname{Re}(\langle e, g\rangle\langle f, h\rangle-\langle e, h\rangle\langle f, g\rangle)|,
\end{aligned}
$$

where the equality holds if and only if $e(x) f(y)-e(y) f(x)=$ $\pm(g(x) h(y)-g(y) h(x))$ for all $x, y \in[a, b]$.

Proof. Note that $e$ and $f$ are complex-valued functions. By using the above definition on $\|e\|^{2},\|f\|^{2}$, and $\langle e, f\rangle$, we have

$$
\begin{aligned}
& \|e\|^{2}\|f\|^{2}-|\langle e, f\rangle|^{2}=\int_{a}^{b} e(x) \overline{e(x)} d x \times \int_{a}^{b} f(x) \\
& \cdot \overline{f(x)} d x-\left|\int_{a}^{b} e(x) \overline{f(x)} d x\right|^{2} \\
& =\iint_{D}(e(x) \overline{e(x)} f(y) \overline{f(y)} \\
& -e(x) \overline{f(x)} e(y) \overline{f(y)}) d x d y=\frac{1}{2} \\
& \cdot \iint_{D}|e(x) f(y)-e(y) f(x)|^{2} d x d y,
\end{aligned}
$$

where $D=\left\{(x, y) \in \mathbb{R}^{2} \mid a \leq x, y \leq b\right\}$. Therefore,

$$
\begin{aligned}
& \|e\|^{2}\|f\|^{2}+\|g\|^{2}\|h\|^{2}-|\langle e, f\rangle|^{2}-|\langle g, h\rangle|^{2}=\frac{1}{2} \\
& \cdot \iint_{D}\left[|e(x) f(y)-e(y) f(x)|^{2}\right. \\
& \left.\quad+|g(x) h(y)-g(y) h(x)|^{2}\right] d x d y \\
& \quad=\frac{1}{2} \iint_{D} \mid(e(x) f(y)-e(y) f(x)) \\
& \quad \pm\left.(g(x) h(y)-g(y) h(x))\right|^{2}
\end{aligned}
$$

$$
\begin{aligned}
& \mp 2 \operatorname{Re}[(e(x) f(y)-e(y) f(x)) \\
& \cdot(\overline{g(x) h(y)-g(y) h(x)})] d x d y \\
& \geq \mp \iint_{D} \operatorname{Re}[(e(x) f(y)-e(y) f(x)) \\
& \cdot(\overline{g(x) h(y)-g(y) h(x)})] d x d y \\
& =\mp 2 \operatorname{Re}\left(\int_{a}^{b} e(x) \overline{g(x)} d x \int_{a}^{b} f(y) \overline{h(y)} d y\right. \\
& \left.-\int_{a}^{b} e(x) \overline{h(x)} d x \int_{a}^{b} f(y) \overline{g(y)} d y\right) \\
& =\mp 2 \operatorname{Re}(\langle e, g\rangle\langle f, h\rangle-\langle e, h\rangle\langle f, g\rangle) .
\end{aligned}
$$

Then the inequality follows, as required.

By a similar argument, we further obtain the following.

Theorem 4. Let $f_{1}, \ldots, f_{m}, h_{1}, \ldots, h_{m}$ be $2 m$ continuous complex-valued functions on $[a, b]$. Then, for $m \geq 2$, one has

$$
\begin{aligned}
\sum_{k=1}^{m}\left\|f_{k}\right\|^{2}\left\|h_{k}\right\|^{2} \geq \sum_{k=1}^{m}\left|\left\langle f_{k}, h_{k}\right\rangle\right|^{2}+\frac{2}{m-1} \\
\cdot \sum_{1 \leq k<l \leq m}\left|\operatorname{Re}\left(\left\langle f_{k}, f_{l}\right\rangle\left\langle h_{k}, h_{l}\right\rangle-\left\langle f_{k}, h_{l}\right\rangle\left\langle h_{k}, f_{l}\right\rangle\right)\right|,
\end{aligned}
$$

where the equality holds if and only if $f_{k}(x) h_{k}(y)-f_{k}(y) h_{k}(x)$ $= \pm\left(f_{l}(x) h_{l}(y)-f_{l}(y) h_{l}(x)\right)$ for all $k, l=1, \ldots, m$ and all $x, y \in[a, b]$.

Remark 5. (i) In this paper, we study the Cauchy-Schwarz inequality by using the real or complex valued functions defined on an interval $[a, b]$. Indeed, it holds for any other continuous functions defined on a measurable set.

(ii) To prove the results, we follow the arguments from $[6,7]$. We remark that, by using the Gramian matrix $G$ defined by

$$
G=\left(\begin{array}{cccc}
\langle e, e\rangle & \langle e, f\rangle & \langle e, g\rangle & \langle e, h\rangle \\
\langle f, e\rangle & \langle f, f\rangle & \langle f, g\rangle & \langle f, h\rangle \\
\langle g, e\rangle & \langle g, f\rangle & \langle g, g\rangle & \langle g, h\rangle \\
\langle h, e\rangle & \langle h, f\rangle & \langle h, g\rangle & \langle h, h\rangle
\end{array}\right),
$$

we can also prove Theorem 1 . In fact, since $G=\left(\begin{array}{ll}A & B \\ B & C\end{array}\right) \geq 0$, it holds that $\left(\begin{array}{l}\operatorname{det} A \operatorname{det} B \\ \operatorname{det} B \operatorname{det} C\end{array}\right) \geq 0$, from which Theorem 1 follows. Further, using $2 n \times 2 n$ Gramian matrix, we generalized the result to Theorem 2. Complex cases are also derived in the same way.

\section{Conflicts of Interest}

The author declares that they have no conflicts of interest. 


\section{Acknowledgments}

This work is supported by AHNSF (1608085MA03) and TLXYRC (2015tlxyrc09).

\section{References}

[1] A.-L. Cauchy, Cours d'Analyse de l'Ecole Royale Polytechnique, I Partie, Analyse Algebrique, Paris, France, 1821.

[2] D. S. Mitrinović, J. E. Pečarić, and A. M. Fink, Classical and New Inequalities in Analysis, Kluwer Academic, Dordrecht, The Netherlands, 1993.

[3] V. Y. Buniakowski, "Sur quelques inegalites concernant les integrales aux differences finies," Mémoires de l'Académie impériale des sciences de St. Pétersbourg, vol. 1, no. 9, pp. 1-18, 1859.

[4] S. S. Dragomir, "A survey on Cauchy-Bunyakovsky-Schwarz type discrete inequalities," JIPAM. Journal of Inequalities in Pure and Applied Mathematics, vol. 4, no. 3, article 63, 142 pages, 2003.

[5] J. C. Kuang, Applied Inequalities, ShanDong Science and Technology Press, Jinan, China, 2004.

[6] N. J. Harvey, "A generalization of the Cauchy-Schwarz inequality involving four vectors," Journal of Mathematical Inequalities, vol. 9, no. 2, pp. 489-491, 2015.

[7] D. Choi, "A generalization of the Cauchy-Schwarz inequality," Journal of Mathematical Inequalities, vol. 10, no. 4, pp. 10091012, 2016. 


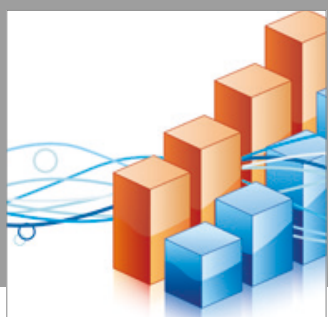

Advances in

Operations Research

vatersals

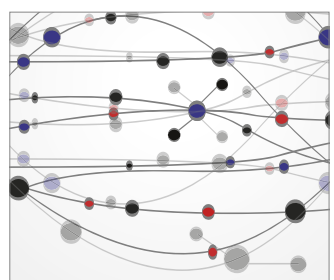

\section{The Scientific} World Journal
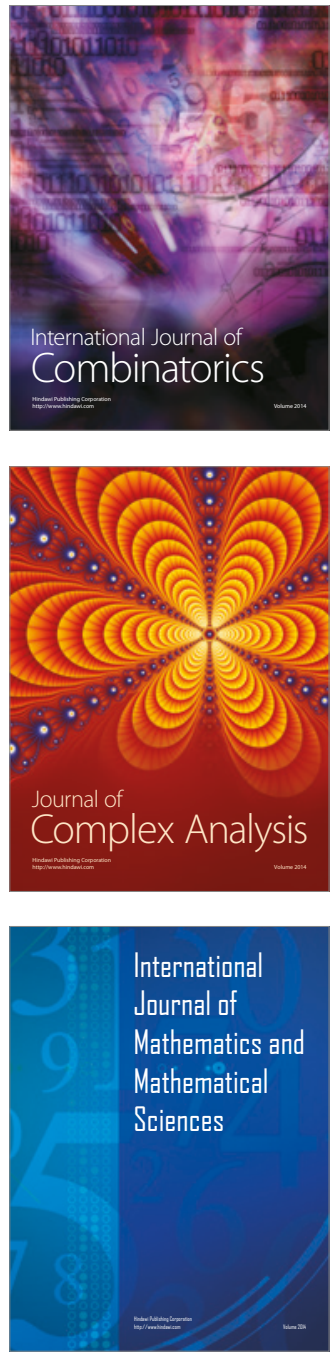
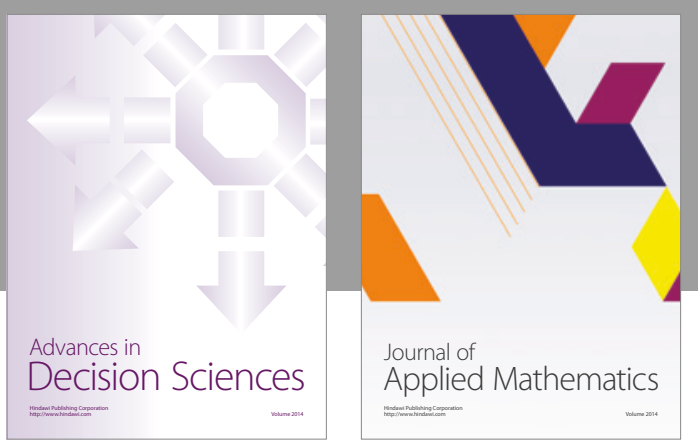

Algebra

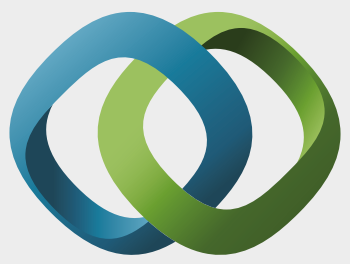

\section{Hindawi}

Submit your manuscripts at

https://www.hindawi.com
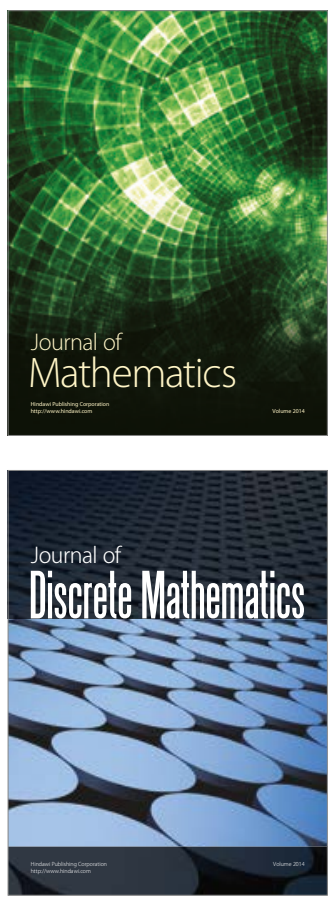

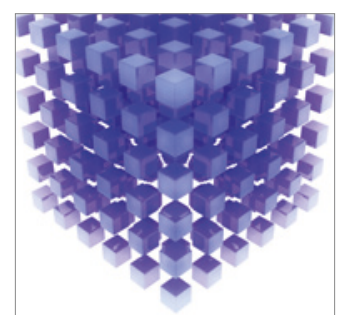

Mathematical Problems in Engineering
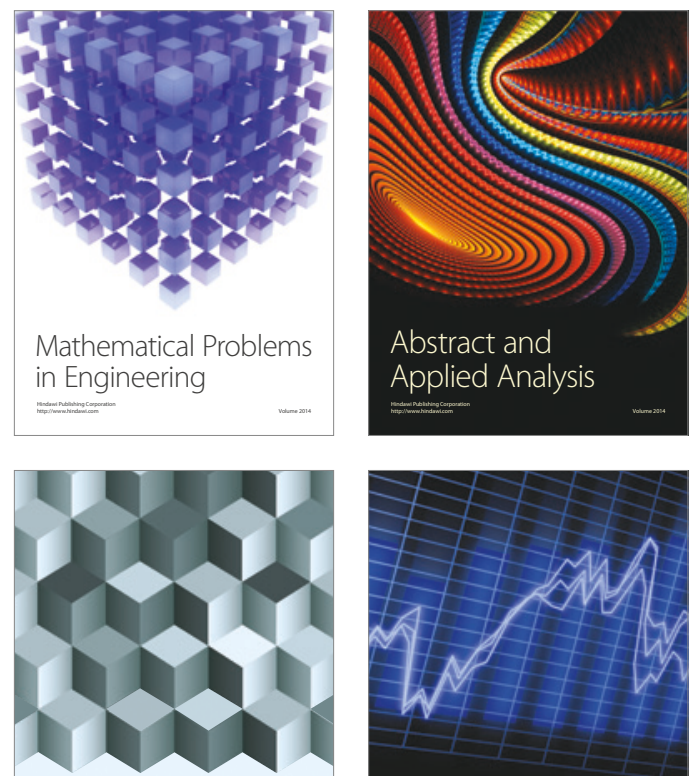

Journal of

Function Spaces

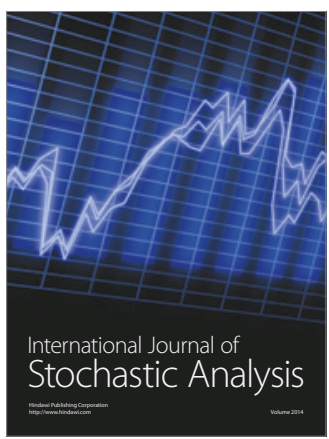

Probability and Statistics
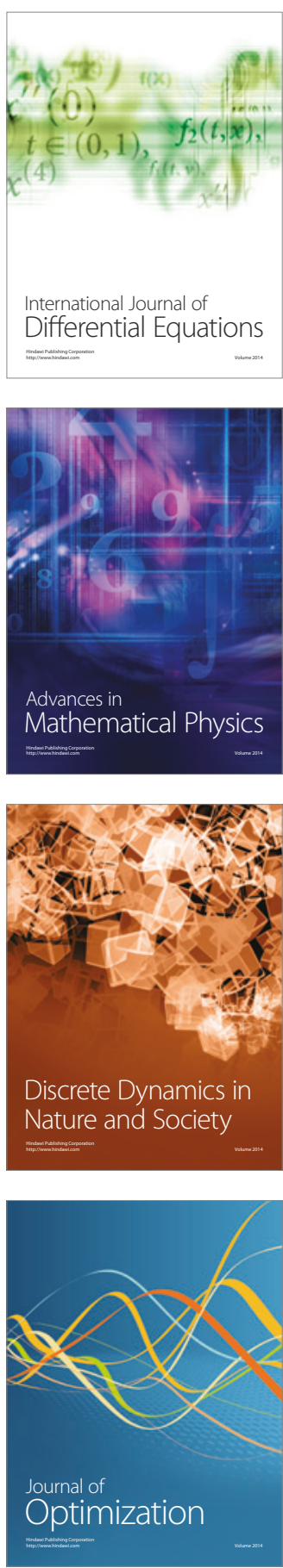